\title{
A mesh-free approach for the simulation of incompressible flows
}

\section{Rammane ${ }^{1}$, S. Mesmoudi ${ }^{1}$, A. Tri ${ }^{2,3}$, B. Braikat ${ }^{1}$, N. Damil ${ }^{1}$}

\section{Laboratoire d'Ingénierie et Matériau, Faculté des Sciences Ben M’sik, Hassan II university of Casablanca, B.P 7955 Sidi} Othman, Casablanca, Morocco.

2. Laboratoire de Mécanique, Faculté des Sciences Ain Chock, Hassan II university of Casablanca, Morocco. 3. Institut Supérieur des Etudes Maritimes (ISEM), Km 7 Route d'El Jadida, Casablanca, Morocco.

\begin{abstract}
:
In this work, we propose to investigate numerically the incompressible flows by the Asymptotic Numerical Method (ANM) with the Moving Least Square (MLS). The mathematical formulation is based on the Navier-Stokes equations written in a strongly formulation to avoid all difficulties of the numerical integration. The used algorithm is developed to investigate the effective of the ANM with the MLS in the strongly formulation.
\end{abstract}

Keywords: MLS, Asymptotic Numerical Method, Navier-Stokes equations, incompressible flows.

\section{Introduction}

Several methods have been proposed to solve several problems of fluid mechanics. The concept of each method differs from one problem to another depending on the difficulties encountered during the discretization or resolution. The cost time has always been an important parameter of these methods. This factor has limited the use of some of these methods and it has motivated others which have been successful in recent years. Among these methods, meshless methods have been developed rapidly as tools of discretization and approximation. Most of these methods have been mainly, attending to solve problems mechanics [1]. Their theoretical foundation allowed the researchers to avoid problems related to the mesh. Indeed, these meshless methods have been proposed to solve the mesh generation problems encountered in classical numerical methods such as finite elements and finite volumes for complex problems. This advantage makes it possible to freely add and delete points of the mesh generated inversely to the finite element method requiring adaptive re-meshing with a high cost time.

Especially designed to solve fluid problems, several meshless methods are developed and used to solve the NavierStokes equations $[2,3]$.

In this work, we propose to investigate numerically the incompressible fluid flows by the ANM with MLS. This algorithm is based on the use of the Asymptotic Numerical Method [4] with MLS [1] as a discretization method. The performance of this algorithm will be tested on an example of backward-facing step [5]. The results obtained using our approach will be compared with those obtained by the Newton-Raphson method with MLS.

\section{Governing equations}

The two-dimensional incompressible flow of a fluid is completely described by the Navier-Stokes equations. If $\Omega$ is the domain occupied by the fluid and $\partial \Omega$ is its boundary, these equations are written in the steady state as follows:

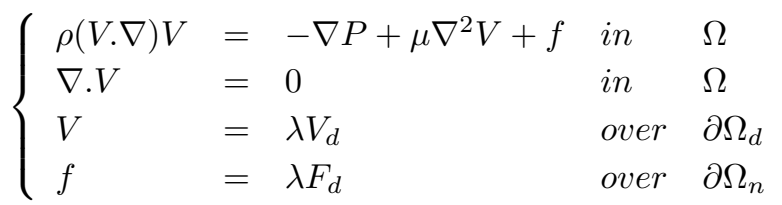

where $\rho$ is the fluid density, $\mu$ is his dynamic viscosity, $V={ }^{T}<u, v>$ is the velocity field, $P$ is the hydrostatic pressure, $V_{d}$ and $F_{d}$ are the Direchlet and Neumann boundary conditions respectively and $\lambda$ is a control parameter. In order to satisfy the incompressibility condition, we introduce the penalty method to express the pressure field versus the speed field. The problem (1) becomes:

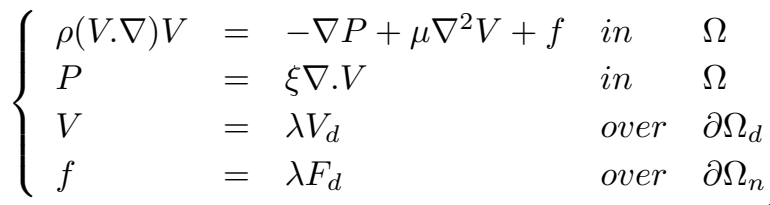

This problem can be writing in a quadratic form (3) for the used algorithm to be easy applied:

$$
\left\{\begin{array}{lllll}
L(V)+G(P)+Q(V, V) & = & \lambda F & \text { in } & \Omega \\
P-\xi D(V) & = & 0 & \text { in } & \Omega \\
V & = & \lambda V_{d} & \text { over } & \partial \Omega_{d}
\end{array}\right.
$$

\section{Resolution strategy}

The problem (3) is a non-linear system that requires numerical resolution techniques such as iterative or NewtonRaphson methods. For that, we propose an algorithm based on the following steps: Development of Taylor series, approximation MLS meshless type followed by a continuation procedure. 


\subsection{Taylor series expansion}

In order to transform the nonlinear problem (3) into a sequence of linear ones, the variables $(V, P, \lambda)$ of (3) are sought in the Taylor series development with respect to a parameter of path " $a$ " defined by the famous arc-length continuation method [4]. The expansion development is defined by:

$$
\left\{\begin{array}{l}
V \\
P \\
\lambda
\end{array}\right\}=\left\{\begin{array}{l}
V_{0} \\
P_{0} \\
\lambda_{0}
\end{array}\right\}+\sum_{i=1}^{i=k}\left\{\begin{array}{c}
V_{i} \\
P_{i} \\
\lambda_{i}
\end{array}\right\}
$$

with $\left(V_{0}, P_{0}, \lambda_{0}\right)$ are the initial considered known variables at each branch of the solution and $\left(V_{i}, P_{i}, \lambda_{i}\right)$ corresponds to the solutions at order $i$. This series development is injected into the problem (3) and by identifying each term according to the powers of the parameter "a", we obtain a succession of linear systems all having the same tangent operator that are easy to solve. These linear systems are defined at each order by:

At order $i=1$ :

$$
\left\{\begin{array}{lll}
L_{t}\left(V_{1}\right)+G\left(P_{1}\right) & = & \lambda_{1} F \\
P_{1}-\xi D\left(V_{1}\right) & = & 0 \\
<V_{1}>\left\{V_{1}\right\}+\lambda_{1}^{2} & = & 1
\end{array}\right.
$$

At order $2 \leq i \leq k$ :

$$
\left\{\begin{array}{lll}
L_{t}\left(V_{i}\right)+G\left(P_{i}\right) & = & \lambda_{i} F+F^{n l} \\
P_{i}-\xi D\left(V_{i}\right) & = & 0 \\
<V_{1}>\left\{V_{i}\right\}+\lambda_{1} \lambda_{i} & = & 0
\end{array}\right.
$$

where $L_{t}(\bullet)=L(\bullet)+Q\left(V_{0}, \bullet\right)+Q\left(\bullet, V_{0}\right)$ and $F^{n l}=-\sum_{r=1}^{i-1} Q\left(V_{r}, V_{i-r}\right)$

\subsection{MLS approximation}

So many physical and mechanical problems in particular have been solved using conventional discretization methods such as the finite element method. These methods often face computational cost difficulties required by adaptive re-meshing to the state of the problem treated especially for complex problems in large deformations. To overcome these disadvantages, meshless methods have been successfully developed. We use among these MLS meshless presented in $[6,7]$ for approximate the unknowns of the problem at any point $M(x, y)$ of support $\Omega_{s}$ as follow:

$$
\left\{\begin{array}{l}
\left\{V_{M}\right\}=\left[\Phi_{V}\right]\left\{V_{s}\right\} \\
P_{M}=\left[\Phi_{P}\right]\left\{P_{s}\right\}
\end{array}\right.
$$

where $\left[\Phi_{V}\right]$ and $\left[\Phi_{V}\right]$ are the matrices with the size $(2 \times 2 N)$ and $(1 \times N)$ respectively which are constructed by the MLS shape function with $N$ is the neighbors in the support do- main $\Omega_{s}$. Taken into account of these approximations and adopting an assembly technique in the whole neighbors for all points in domain, we obtain the compact problem described by:

At order $i=1$ :

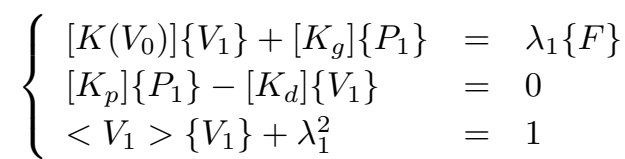

At order $2 \leq i \leq k$ :

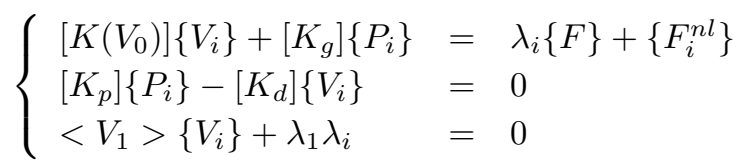

To solve the obtained problem verified by the speed and pressure fields. By the penalty method, we transform this problem into a problem with the velocity as unknown as follow:

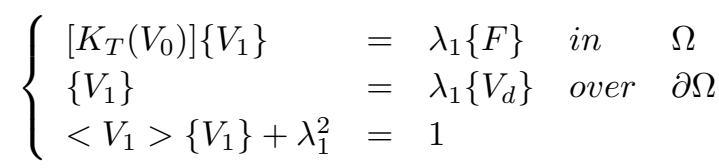

At order $2 \leq i \leq k$ :

$$
\left\{\begin{array}{llll}
{\left[K_{T}\left(V_{0}\right)\right]\left\{V_{i}\right\}} & =\lambda_{i}\{F\}+\left\{F_{i}^{n l}\right\} & \text { in } & \Omega \\
\left\{V_{i}\right\} & =\lambda_{i}\left\{V_{d}\right\} & \text { over } & \partial \Omega \\
<V_{1}>\left\{V_{i}\right\}+\lambda_{1} \lambda_{i} & = & 0 &
\end{array}\right.
$$

\subsection{Continuation technique}

For the calculation of the whole solution, we use the technique of continuation [4] which consists in estimating the range of validity of the series of Taylor by the criterion $a_{\max }$ following:

$$
a_{\max }=\left(\epsilon \frac{\left\|\left\{V_{1}\right\}\right\|}{\left\|\left\{V_{k}\right\}\right\|}\right)^{\frac{1}{k-1}}
$$

where $\epsilon$ is a tolerance parameter and $\|\bullet\|$ is the Euclidean norm. In addition, this technique consists in considering the point $\left(\left\{V\left(a_{\max }\right)\right\}, \lambda\left(a_{\max }\right)\right.$ as being the starting point $\left(\left\{V_{0}\right\}, \lambda_{0}\right)$ of the following branch. The whole solution of the problems $(10,11)$ is determined branch by branch $[8,6,7]$.

\section{4 numerical application}

To evaluate the efficiency of the algorithm developed in the previous sections, we propose a numerical test of the flow problem in a backward-facing step [5]. The geometry and the boundary conditions are presented in figure (1) 
of the following characteristics: $l=1 \mathrm{~m}, b=2.5 \mathrm{~m}$ and , $L=18 \mathrm{~m}$. The Reynolds number is defined by $R e=\frac{\lambda u_{d} \rho}{\mu}$ with $\rho=1 \mathrm{Kg} / \mathrm{m}^{3}$ and $\mu=0.1 \mathrm{Kg} /(\mathrm{m} . \mathrm{s})$.

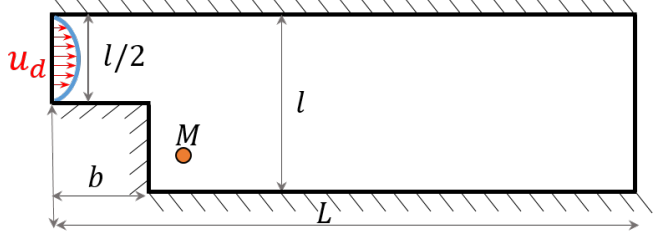

Figure 1: Geometric of backward-facing step

We choose a truncation order of series $k=15$ and a tolerance parameter $\epsilon=10^{-6}$. The MLS shape functions presented in $[6,7]$ are used with the radius $h$ of support domain is chosen in this study as follows:

$$
h=d \frac{\sqrt{A}}{\sqrt{N_{d}}-1} \quad d=3
$$

with $A$ is the area of the estimated domain and $N_{d}=7081$ is the number of nodes covered by the domain with the area of $A$. The results obtained are compared with those obtained using a predictor-corrector algorithm based on the coupling of the Newton-Raphson method with MLS method.

In figure 2, horizontal and vertical velocity profiles versus Reynolds number at the point $M(3.5,0.25)$ computed with the present approach are compared with those given by the Newton-Raphson method with also MLS. The results are in good agreement with a saving of cost time in terms of tangent matrix inversions with our approach (ANM with MLS 20 inversions and Newton-Raphson with MLS 2624 inversions ).
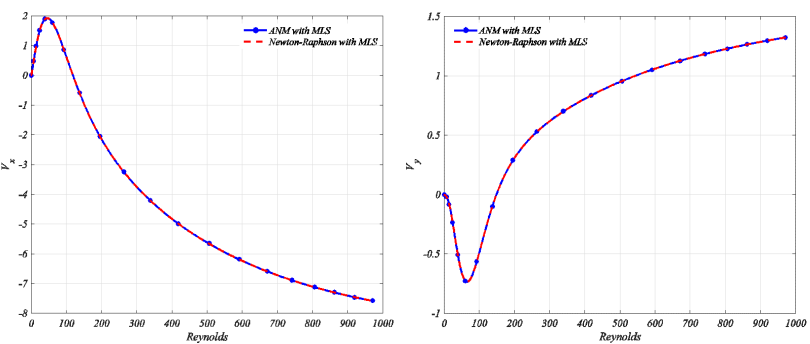

Figure 2: Evolution of the $\mathrm{x}$-component and $\mathrm{y}$-component of velocity versus Reynolds number

Streamlines are plotted in figures 3 and 4 for Reynolds numbers equal to 94 and 924 respectively, showing the vortexflows in the backward-facing step. This vortex-flows are coherent with those found in the literature [5].

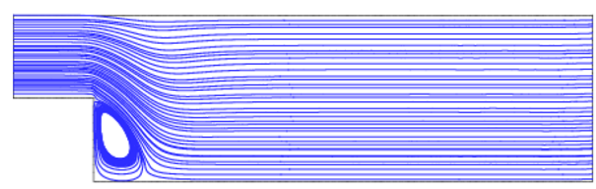

Figure 3: Streamlines for the backward-facing at $R e=94$

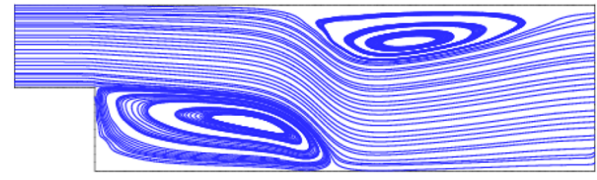

Figure 4: Streamlines for the backward-facing at $R e=924$

\section{Conclusion}

In this work, we have proposed and developed a meshless approach based on the Asymptotic Numerical Method for solving the Navier-Stokes equations in the two-dimensional stationary case. The MLS method has been used in the ANM method. According to these obtained results, we note that the used algorithm is effective to solve the incompressible fluid equations. The work is in progress to extend this technique to compressible fluid flows.

\section{References}

[1] G.-R. Liu, Y.-T. Gu, An introduction to meshfree methods and their programming, Springer Science \& Business Media, 2005.

[2] C. Felter, J. H. Walther, C. Henriksen, Moving least squares simulation of free surface flows, Computers \& Fluids 91 (2014) 47-56.

[3] G. Bourantas, V. Loukopoulos, H. Chowdhury, G. Joldes, K. Miller, S. Bordas, An implicit potential method along with a meshless technique for incompressible fluid flows for regular and irregular geometries in $2 \mathrm{~d}$ and $3 \mathrm{~d}$, Engineering Analysis with Boundary Elements 77 (2017) 97-111.

[4] B. Cochelin, N. Damil, M. Potier-Ferry, Méthode asymptotique numérique, Hermes Lavoissier, 2007.

[5] J. Cadou, M. Potier-Ferry, B. Cochelin, N. Damil, Anm for stationary navier-stokes equations and with petrovgalerkin formulation, International Journal for Numerical Methods in Engineering 50 (4) (2001) 825-845.

[6] S. Mesmoudi, A. Timesli, B. Braikat, H. Lahmam, H. Zahrouni, A 2d mechanical-thermal coupled model to simulate material mixing observed in friction stir welding process, Engineering with Computers 33 (4) (2017) 885-895.

[7] Y. Belaasilia, A. Timesli, B. Braikat, M. Jamal, A numerical mesh-free model for elasto-plastic contact problems, Engineering Analysis with Boundary Elements 82 (2017) 68-78.

[8] A. Timesli, B. Braikat, H. Lahmam, H. Zahrouni, A new algorithm based on moving least square method to simulate material mixing in friction stir welding, Engineering Analysis with Boundary Elements 50 (2015) 372-380. 